\title{
Boundedness and asymptotic stability for delayed equations of logistic type
}

\author{
Teresa Faria \\ Departamento de Matemática, Faculdade de Ciências/CMAF, \\ Universidade de Lisboa, R. Ernesto Vasconcelos, 1749-016 Lisboa, \\ Portugal (tfaria@lmc.fc.ul.pt)
}

\section{Eduardo Liz}

Departamento de Matemática Aplicada II, ETSI Telecomunicación Universidad de Vigo, Campus Marcosende, 36280 Vigo, Spain (eliz@dma.uvigo.es)

(MS received 13 November 2002; accepted 28 March 2003)

For a scalar Lotka-Volterra-type delay equation $\dot{x}(t)=b(t) x(t)\left[1-L\left(x_{t}\right)\right]$, where $L: C([-r, 0] ; \mathbb{R}) \rightarrow \mathbb{R}$ is a bounded linear operator and $b$ a positive continuous function, sufficient conditions are established for the boundedness of positive solutions and for the global stability of the positive equilibrium, when it exists. Special attention is given to the global behaviour of solutions for the case of $L$ a positive linear operator. The approach used for this situation is applied to address the global asymptotic stability of delayed logistic models in the more general form $\dot{x}(t)=b(t) x(t)\left[a(t)-L\left(t, x_{t}\right)\right]$, with $L(t, \cdot)$ being linear and positive.

\section{Introduction}

Differential equations with time delays have been extensively considered as models for many problems in biology and other sciences, with particular emphasis on population dynamics models. Among them, a quite simple equation is the Hutchinson equation

$$
\dot{y}(t)=b y(t)[1-y(t-r) / K],
$$

the delayed version of the logistic equation used to study the growth of a single species population, where the delay $r>0$ is introduced to take into account the maturation period of the species. The study of global asymptotic stability conditions for the positive equilibrium $K$ of (1.1) goes back at least to the work of Wright [14]. By the change of variables $x(t)=-1+y(r t) / K$, the Hutchinson equation becomes

$$
\dot{x}(t)=-\alpha x(t-1)[1+x(t)],
$$

with $\alpha=b r$, usually known as the Wright equation. As it is well known, Wright proved that the condition $0<\alpha \leqslant \frac{3}{2}$ implies that all solutions $x(t)$ of $(1.2)$ such that $x(0)>-1$ converge to zero as $t \rightarrow \infty$. This $\frac{3}{2}$-stability condition was extended to the case of variable coefficients, that is, to the equation

$$
\dot{x}(t)=-b(t) x(t-1)[1+x(t)]
$$


by So and $\mathrm{Yu}$ [11], who proved that condition $\int_{t-1}^{t} b(s) \mathrm{d} s \leqslant \frac{3}{2}$ for large $t$ ensures the global asymptotic stability of the zero solution in (1.3) under the additional condition $\int_{0}^{+\infty} b(s) \mathrm{d} s=+\infty$ (see also [1]). A remarkable extension can be found in [12], where a $\frac{3}{2}$-stability result is obtained for a more general delayed nonlinear logistic-type equation. For more discussions and related results on $\frac{3}{2}$-type stability conditions, see $[6, \S 4.5]$.

There is an extensive literature dealing with delayed logistic and Lotka-Volterratype equations. Among them and related to our present work, we refer the reader to the books of Gopalsamy [2] and Kuang [6], and the works of Györi [3], Haddock and Kuang [4], Kuang and Smith [7], Seifert [10] and So and $\mathrm{Yu}[11,12]$, to mention only a few, and the references therein.

Typically, bound conditions involving the size of the coefficients and the size of the delays, such as $\frac{3}{2}$-type conditions, are used to force oscillatory solutions to zero as $t \rightarrow \infty$. Rather than considering a $\frac{3}{2}$-type condition, another approach is to impose that the equation has an undelayed negative feedback term that dominates the delayed part. The present work fits in this setting: in a quite general framework of delayed logistic models, we assume a theoretical condition of undelayed dominance, which enables us to deal with oscillatory solutions. Furthermore, we do not make use of a Lyapunov functional or Razumikhin methods, which are the techniques usually employed in the literature.

In the present paper, we first address the global behaviour of positive solutions of a general delayed logistic equation with an autonomous linearity,

$$
\dot{x}(t)=b(t) x(t)\left[1-L\left(x_{t}\right)\right],
$$

where $L: C([-r, 0] ; \mathbb{R}) \rightarrow \mathbb{R}$ is a positive linear operator. The techniques used for (1.4) are extended to the study of more general equations, which include both the case of equations with non-autonomous linearities in the form

$$
\dot{x}(t)=b(t) x(t)\left[a(t)-L\left(t, x_{t}\right)\right],
$$

where $L(t, \cdot), t \in \mathbb{R}$, are positive linear operators, and the case of (1.4) with a nonpositive bounded linearity $L$. We emphasize that, for this latter situation, there are some interesting open problems even for the simpler case of discrete delays $($ see $[3, \S 5])$.

We now set some standard notation that will be used throughout the paper. A linear functional $L: C([a, b] ; \mathbb{R}) \rightarrow \mathbb{R}$ is said to be positive if $L(\varphi) \geqslant 0$ whenever $\varphi \geqslant 0$. A solution $x(t)$ is said to be bounded below from zero on an interval $I$ if there is a positive constant $c$ such that $x(t) \geqslant c$ for all $t \in I$. If $x(t)$ is defined for $t \geqslant 0$, we say that $x(t)$ is oscillatory if it is not eventually zero and it has arbitrarily large zeros; otherwise, it is called non-oscillatory; $x(t)$ is called oscillatory (respectively, non-oscillatory) around $x_{*}$ if $x(t)-x_{*}$ is oscillatory (respectively, non-oscillatory).

The present paper is organized as follows. In $\S 2$, the case of (1.4) with $L$ being a positive linear operator is considered. We establish sufficient conditions for the global stability of the positive equilibrium $x_{*}$. In order to guarantee that oscillatory solutions around $x_{*}$ go to $x_{*}$ as $t$ goes to $\infty$, we assume hypothesis (H2) in $\S 2$, which states that $L(\varphi)>0$ for all $\varphi \in C([-r, 0] ; \mathbb{R})$ such that $\sup _{\theta \in[-r, 0]}|\varphi(\theta)|=\varphi(0)>0$. This means that the value $\varphi(0)$ of a function $\varphi \in C([-r, 0] ; \mathbb{R})$ carries some weight to the value of its image $L(\varphi)$, or, in other words, that $L$ has a kind of undelayed 
component that dominates the delayed part. Under the same hypothesis (H2), which is easy to verify in practice, and by studying the asymptotic behaviour of solutions as $t \rightarrow-\infty$, we prove that $x_{*}$ is the only global solution that is bounded and bounded below from zero. Although, in biological terms, only the existence and asymptotic behaviour in the future seem to be relevant, the existence of a persistent and bounded global solution has often been analysed in the case of ordinary differential equations (ODEs) modelling the growth of a single population, and also in the framework of models with delays.

The non-autonomous linearity case in (1.5) is addressed in $\S 3$. Equations (1.5) are assumed to be a perturbation of (1.4), in the sense that we suppose that the limit of $a(t)-L(t, \varphi)$ has the form $a_{0}-L(\varphi)$ as $t \rightarrow \infty$, with $a_{0}>0$ and $L: C \rightarrow \mathbb{R}$ a positive linear operator. By using the techniques in the previous section, general sufficient conditions are given in order to ensure that all the positive solutions tend to the positive equilibrium of $\dot{x}(t)=b(t) x(t)\left[a_{0}-L\left(x_{t}\right)\right]$ as $t \rightarrow \infty$.

In $\S 4$, we consider (1.4) with $L$ no longer positive but a general bounded linear operator. Again, some of the techniques developed in $\S 2$ are used to establish conditions that are sufficient to guarantee that positive solutions are defined and bounded on $[0, \infty)$, as well as that the positive equilibrium (if it exists) is globally asymptotically stable.

Throughout the paper, we illustrate the application of our results by considering several scalar population models with discrete or distributed delays.

\section{Autonomous positive linearity}

Let $C=C([-r, 0] ; \mathbb{R})$ be the space of continuous functions from $[-r, 0]$ to $\mathbb{R}, r>0$, equipped with the sup norm $|\varphi|_{C}=\max _{-r \leqslant \theta \leqslant 0}|\varphi(\theta)|$. In the phase space $C$, consider the scalar functional differential equation (FDE)

$$
\dot{x}(t)=b(t) x(t)\left[1-L\left(x_{t}\right)\right],
$$

where $b: \mathbb{R} \rightarrow \mathbb{R}$ is a continuous function such that $0<\beta_{0} \leqslant b(t) \leqslant \beta^{0}$ for all $t \in \mathbb{R}, \beta_{0}, \beta^{0}$ constants, and $L: C \rightarrow \mathbb{R}$ is a non-zero positive linear operator. Since $L$ is positive, i.e. $L(\varphi) \geqslant 0$ for all $\varphi \in C, \varphi \geqslant 0$, then $L$ is bounded and there is a non-decreasing function $\eta:[-r, 0] \rightarrow \mathbb{R}$ such that

$$
L(\varphi)=\int_{-r}^{0} \varphi(\theta) \mathrm{d} \eta(\theta), \quad \varphi \in C
$$

furthermore,

$$
\|L\|=\int_{-r}^{0} \mathrm{~d} \eta(\theta)=\eta(0)-\eta(-r)>0 .
$$

With $x_{*}=l^{-1}$, where $l:=L(1)=\|L\|$, equation (2.1) reads as

$$
\dot{x}(t)=b(t) x(t) L\left(x_{*}-x_{t}\right) .
$$

For $\varphi \in C$, we consider the initial-value problem (IVP) (2.1) with initial condition

$$
x_{0}=\varphi \text {. }
$$


We denote its solution by $x(\varphi)(t)$ or simply $x(t)$. Due to the biological interpretation of model (2.1), we will always consider admissible initial conditions $\varphi \in C$ such that

$$
\varphi(\theta) \geqslant 0, \quad \theta \in[-r, 0), \quad \varphi(0)>0 .
$$

By the change of variables $y=\left(x-x_{*}\right) / x_{*}$, equation (2.1) is written in the form

$$
\dot{y}(t)=-x_{*} b(t)(1+y(t)) L\left(y_{t}\right)
$$

or, equivalently,

$$
\dot{y}(t)=-x_{*} b(t)(1+y(t)) \int_{t-r}^{t} y(s) \mathrm{d} \eta(s-t) .
$$

This equation is a very particular case of the scalar equation

$$
\dot{y}(t)=-(1+y(t)) \sum_{i=1}^{n} \int_{t-r(t)}^{t} f_{i}(t, y(s)) \mathrm{d} \mu_{i}(t, s)
$$

considered by Kuang [5,6] and So and $\mathrm{Yu}$ [12]. If we take $n=1, r(t)=r$, $\mu(t, s):=\mu_{1}(t, s)=x_{*} \eta(s-t)$ and $f_{1}(t, y)=b(t) y$ for all $t, s, y \in \mathbb{R}$, then equation (2.6) becomes (2.5). We note that $\mu(t, s)$ is non-decreasing in $s$ for each $t$. Assumptions (H1)-(H4) in [5], [6, § 4.3] and [12] are satisfied, except that $\mu(t, s)$ is not necessarily a continuous function with respect to $t$. Nevertheless, one can verify that, for this case, the results in $[5,6,12]$ hold without the continuity of $t \mapsto \mu(t, s)$, since $s \mapsto \eta(s-t) \in B V([t-r, t]), t \in \mathbb{R}$, where $B V([a, b])$ is the space of the functions of bounded variation in $[a, b]$. Therefore, we have existence and uniqueness of solutions of (2.1).

In the following, as well as the above, $x_{*}$ denotes both the constant and the constant function in $C$.

TheOrem 2.1. Consider equation (2.1), where the following hold.

(H1) We have

(i) $b: \mathbb{R} \rightarrow \mathbb{R}$ is a continuous function and there are constants $\beta_{0}$, $\beta^{0}$ such that $0<\beta_{0} \leqslant b(t) \leqslant \beta^{0}$ for all $t \in \mathbb{R}$;

(ii) $L: C \rightarrow \mathbb{R}$ is a positive linear operator with $l:=\|L\|>0$.

Define $x_{*}=l^{-1}$ and consider the IVPs (2.1), (2.2), with solution $x(\varphi)(t)$. Then the following results hold.

(i) $x(\varphi)(t)$ is defined for $t \geqslant 0$, bounded below from zero and bounded on $[0, \infty)$.

(ii) If $x(\varphi)(t)$ is non-oscillatory around $x_{*}$, then $x(\varphi)(t) \rightarrow x_{*}$ as $t \rightarrow \infty$.

(iii) Moreover, if $x(\varphi)(t)$ is oscillatory around $x_{*}$ and

$$
\int_{t-r}^{t} b(\tau) \mathrm{d} \tau \leqslant \frac{3}{2} \text { for large } t
$$

then $x(\varphi)(t) \rightarrow x_{*}$ as $t \rightarrow \infty$. 
Proof. By the change of variables $y=\left(x-x_{*}\right) / x_{*}$, we consider (2.1) written in the form (2.4). Lemma 2.1 and theorem 3.1 of [5] imply (i) and (ii). Statement (iii) follows from theorem 3.1 in [12], since, for $\mu(t, s)=x_{*} \eta(s-t)$, where

$$
L(\varphi)=\int_{-r}^{0} \varphi(\theta) \mathrm{d} \eta(\theta), \quad \varphi \in C
$$

condition

$$
\int_{t-r}^{t}\left(\int_{\tau-r}^{\tau} b(\tau) \mathrm{d} \mu(\tau, s)\right) \mathrm{d} \tau \leqslant \frac{3}{2}
$$

in $[12]$ is equivalent to

$$
x_{*}(\eta(0)-\eta(-r)) \int_{t-r}^{t} b(\tau) \mathrm{d} \tau=\int_{t-r}^{t} b(\tau) \mathrm{d} \tau \leqslant \frac{3}{2} .
$$

REMARK 2.2. We note that the ' $\frac{3}{2}$-condition' (2.7) is an improvement of the assumption in [5] and [6, theorem 3.2, p. 129], where the same result was proven under $\int_{t-r}^{t} b(\tau) \mathrm{d} \tau \leqslant 1$ for large $t$.

The next results shows that the conclusion in theorem 2.1 (iii) is still true if (2.7) is replaced by another condition (H2) stated below.

TheOREm 2.3. Assume (H1) and the following hold.

(H2) For all $\varphi \in C$ such that $|\varphi|_{C}=\varphi(0)>0$, then $L(\varphi)>0$.

Then the solutions $x(t)$ of the IVPs (2.1), (2.2) satisfy $x(t) \rightarrow x_{*}$ as $t \rightarrow \infty$, where $x_{*}=l^{-1}$.

Proof. From theorem 2.1, it is sufficient to consider the case of $x(t)$ oscillatory around $x_{*}$. Effect the change of variables $y=\left(x-x_{*}\right) / x_{*}$, let $y(t)$ be an oscillatory solution of (2.4) and define

$$
\liminf _{t \rightarrow \infty} y(t)=-v, \quad \limsup _{t \rightarrow \infty} y(t)=u .
$$

Clearly, $0 \leqslant v<1,0 \leqslant u<\infty$. Fix $\varepsilon>0$ and let $T>0$ be such that

$$
-v-\varepsilon \leqslant y(t) \leqslant u+\varepsilon, \quad t>T \text {. }
$$

We first consider the case $v \leqslant u$. Consider a sequence $\left(t_{n}\right)$ such that $t_{n}-r>T$, $\dot{y}\left(t_{n}\right)=0, y\left(t_{n}\right)>0, y\left(t_{n}\right) \rightarrow u$ and $t_{n} \rightarrow \infty$ as $n \rightarrow \infty$. From (2.4), it follows that $L\left(y_{t_{n}}\right)=0$.

Since both $y(t)$ and $\dot{y}(t)$ are uniformly bounded on $[0, \infty), y(t)$ is uniformly continuous on $[0, \infty)$. This implies that the sequence $\left(y_{t_{n}}\right) \subset C$ is uniformly bounded and equicontinuous, and thus there is a subsequence, still denoted by $\left(y_{t_{n}}\right)$, that converges to a function $\varphi$ on $C$. From $L\left(y_{t_{n}}\right)=0$, we get $L(\varphi)=0$. On the other hand, equation (2.8) and $v \leqslant u$ imply that $\left|y_{t_{n}}\right|_{C} \leqslant u+\varepsilon$. By letting $n \rightarrow \infty$ and since $\varepsilon>0$ is arbitrary, we conclude that $|\varphi|_{C} \leqslant u=\varphi(0)$. From (H2), it follows that $u=0$, hence $v=0$, and the conclusion follows. 
If $v \geqslant u$, we consider a sequence $\left(t_{n}\right)$ as above, except that now $y\left(t_{n}\right)<0$ and $y\left(t_{n}\right) \rightarrow-v$ as $n \rightarrow \infty$, and, in a similar way, we deduce that $v=0$, implying also $u=0$.

REMARK 2.4. Roughly speaking, assumption (H2) means that the non-decreasing function $\eta:[-r, 0] \rightarrow \mathbb{R}$ defining the operator $L$ has more weight near zero. This is the case when $L$ has a non-delayed part that dominates the delayed part. In order to clarify this statement, we consider an important particular case of (2.1). Suppose that

$$
L(\varphi)=b_{0} \varphi(0)+L_{0}(\varphi)
$$

where $L_{0}: C \rightarrow \mathbb{R}$ is a positive linear operator and $b_{0}>0$. If $b_{0}>\left\|L_{0}\right\|$, then, for $\varphi \in C$ such that $\varphi(0)>0$, we have

$$
L(\varphi) \geqslant b_{0} \varphi(0)-\left\|L_{0}\right\||\varphi|_{C},
$$

and consequently (H2) holds. In the general framework of any positive linear operator $L$ from $C$ to $\mathbb{R}$, we note that (H2) is fulfilled if $L$ satisfies the following condition:

$$
\left.\begin{array}{c}
\text { there is a constant } d \in(0,1) \text { such that if } \varphi \in C, \\
\varphi(0)>0 \text { and } L(\varphi)=0, \text { then } \varphi(0) \leqslant d|\varphi|_{C} .
\end{array}\right\}
$$

For the above example, condition (2.10) is satisfied with $d=\left\|L_{0}\right\| / b_{0}$. Equation (2.1) with $L$ given by (2.9) has been considered by many authors. We remark that in [6, pp. 34-35], the global stability of $x_{*}$, which follows here from theorem 2.3, was proven for a more general setting that does not require $L_{0}$ to be positive. The case of (2.1) with $L$ a non-positive linear bounded operator will be addressed in $\S 4$.

ExAmple 2.5. Consider again $L$ as in (2.9), but assume now that $b_{0}=\left\|L_{0}\right\|>0$. Write $L_{0}(\varphi)=\int_{-r}^{0} \varphi(\theta) \mathrm{d} \eta_{0}(\theta)$ for $\varphi \in C$, where $\eta_{0}:[-r, 0] \rightarrow \mathbb{R}$ is non-decreasing. If we further impose that there exists $\varepsilon \in(0, r)$ such that $\eta_{0}(\theta)<\eta_{0}(0)$ for $-\varepsilon<$ $\theta<0$, then condition (H2) is satisfied. In fact, let $\varphi \in C$ with $|\varphi|_{C}=\varphi(0)>0$. Choose $\xi \in(-\varepsilon, 0)$ such that $\varphi(\theta)>0$ for $\theta \in[\xi, 0]$. Then

$$
\begin{aligned}
L(\varphi) & \geqslant b_{0} \varphi(0)+\int_{-r}^{\xi} \varphi(\theta) \mathrm{d} \eta_{0}(\theta) \\
& \geqslant b_{0} \varphi(0)-\varphi(0)\left(\eta_{0}(\xi)-\eta_{0}(-r)\right) \\
& >\varphi(0)\left(b_{0}-\left\|L_{0}\right\|\right) \\
& =0 .
\end{aligned}
$$

As a concrete example of the above situation, consider the equation

$$
\dot{x}(t)=b(t) x(t)\left[1-x(t)-\int_{-1}^{0} x(t+\theta) \mathrm{d} \theta\right],
$$

where $b(t)$ is as in (H1) (i). Thus (H2) holds for $L(\varphi)=\varphi(0)+\int_{-1}^{0} \varphi(\theta) \mathrm{d} \theta$. We can also use this example to show that (2.10) is strictly stronger than (H2). Let $\varepsilon \in(0,1)$, and define $\varphi_{\varepsilon}:[-1,0] \rightarrow \mathbb{R}$ by

$$
\varphi_{\varepsilon}(x)= \begin{cases}-1-\varepsilon & \text { if } x \in\left[-1, t_{0}\right], \\ \frac{(2+\varepsilon)^{2}}{2 \varepsilon} x+1 & \text { if } x \in\left(t_{0}, 0\right],\end{cases}
$$


where $t_{0}=-2 \varepsilon /(2+\varepsilon) \in(-1,0)$. Clearly, we have $\left|\varphi_{\varepsilon}\right|_{C}=1+\varepsilon, L\left(\varphi_{\varepsilon}\right)=0$ and $\varphi_{\varepsilon}(0)=1>0$. If $(2.10)$ is fulfilled, we get $1 \leqslant d(1+\varepsilon)$. Since $\varepsilon>0$ is arbitrary, then $1 \leqslant d$; a contradiction. Therefore, for $L$ as above, condition (2.10) fails.

Inspired by the approach of [9], in the next lemma we give an estimate for the future of the distance between a solution $x(t)$ and $x_{*}$, in terms of that distance in the past.

Lemma 2.6. Assume (H1) (ii) and (H2) and that $b: \mathbb{R} \rightarrow \mathbb{R}$ is a positive continuous function. Define $x_{*}=l^{-1}$. Let $x(t)=x(\varphi)(t)$ be the solution of (2.1), (2.2), suppose that $x(t)$ is defined on $I=(a, \infty)(a \in \mathbb{R}$ or $a=-\infty)$ and there exist $K>0$ and $t_{0}>a+r$ such that $x(t)$ satisfies

$$
\left|x(t)-x_{*}\right| \leqslant K \quad \text { for } t \in\left(a, t_{0}\right] .
$$

Then

$$
\left|x(t)-x_{*}\right| \leqslant K \quad \text { for } t \in\left[t_{0}, \infty\right)
$$

Proof. Suppose that there exists $t_{1}>t_{0}$ such that $\left|x\left(t_{1}\right)-x_{*}\right|>K$. Define

$$
T=\min \left\{t \in\left[t_{0}, t_{1}\right]:\left|x(t)-x_{*}\right|=\sup _{t_{0} \leqslant s \leqslant t_{1}}\left|x(s)-x_{*}\right|\right\} .
$$

Then $T \in\left(t_{0}, t_{1}\right]$ and

$$
\left|x(s)-x_{*}\right|<\left|x(T)-x_{*}\right| \text { for } t_{0}<s<T .
$$

Since (2.12) implies $\left|x(s)-x_{*}\right| \leqslant K<\left|x(T)-x_{*}\right|$, for $s \in\left(a, t_{0}\right]$, in particular, we obtain

$$
\left|x_{T}(\theta)-x_{*}\right|<\left|x(T)-x_{*}\right|, \quad-r \leqslant \theta<0 .
$$

If $x(T)-x_{*}>0$, then, for $a<s<T$, we have

$$
x(s)-x_{*}<x(T)-x_{*},
$$

and we deduce that $\dot{x}(T) \geqslant 0$. But this leads to a contradiction, since (H2) implies that

$$
\dot{x}(T)=-b(T) x(T) L\left(x_{T}-x_{*}\right)<0 .
$$

The case $x(T)-x_{*}<0$ is treated in a similar way.

REMARK 2.7. Note that the proof above is still valid if we replace (H2) by the slightly weaker hypothesis

$$
\text { if } \varphi \in C \text { and }|\varphi(\theta)|<\varphi(0) \text { for } \theta \in[-r, 0) \text {, then } L(\varphi)>0 \text {. }
$$

We now study the behaviour of global solutions of (2.1), i.e. solutions $x(t)$ defined on $\mathbb{R}$.

Lemma 2.8. Let $x_{*}=l^{-1}$. Assume (H1) and (H2). If $x(t)=x(\varphi)(t)$ is a global solution of (2.1) that is bounded below from zero and bounded on $(-\infty, \infty)$, then $x(t) \rightarrow x_{*}$ as $t \rightarrow-\infty$. 
Proof. Effect the change of variables $y=\left(x-x_{*}\right) / x_{*}$ and consider (2.4). Let $y(t)$ be a solution of $(2.4)$ on $\mathbb{R}$, with $-1<m \leqslant y(t) \leqslant M$ for all $t \in \mathbb{R}$.

If $y(t)<0$, for all $t \in\left(-\infty, T_{0}\right]$ and some $T_{0} \in \mathbb{R}$, the positivity of $L$ implies that $y(t)$ is non-decreasing on $\left(-\infty, T_{0}\right]$, and hence there is $c=\lim _{t \rightarrow-\infty} y(t)<0$. For $t \leqslant T_{1},-T_{1}>0$ large, we have $L\left(y_{t}\right) \leqslant \frac{1}{2} l c$, and thus $\dot{y}(t) \geqslant-\frac{1}{2}(1+y(t)) \beta_{0} c$, implying that

$$
1+y\left(T_{1}\right) \geqslant(1+y(t)) \exp \left(-\frac{1}{2} \beta_{0} c\left(T_{1}-t\right)\right), \quad t \leqslant T_{1} .
$$

The right-hand side of the above inequality tends to $\infty$ as $t \rightarrow-\infty$; a contradiction. A similar argument shows that the situation $y(t)>0, t \in\left(-\infty, T_{0}\right]$ for some $T_{0} \in \mathbb{R}$ also cannot happen. Hence $y(t)$ is oscillatory near $-\infty$, i.e. $y(-t)$ is oscillatory. The proof that $\lim _{t \rightarrow-\infty} y(t)=0$ is analogous to the proof of theorem 2.3, replacing $y(t)$ by the function $z(t)=y(-t)$, so we omit it.

TheOREM 2.9. Assume (H1) and (H2). Then the only solution of (2.1) that is defined on $\mathbb{R}$, bounded below from zero and bounded, is the constant solution $x(t)=x_{*}$, where $x_{*}=l^{-1}$.

Proof. Let $x(t)$ be a global solution of (2.1) that is bounded below from zero and bounded. By lemma 2.8, $\lim _{t \rightarrow-\infty} x(t)=x_{*}$. Thus, for any arbitrary $\varepsilon>0$, there exists $t_{0}=t_{0}(\varepsilon)$ such that $\left|x(t)-x_{*}\right| \leqslant \varepsilon$, for $t \leqslant t_{0}$. On the other hand, from lemma 2.6, we have $\left|x(t)-x_{*}\right| \leqslant \varepsilon$ for all $t \in \mathbb{R}$, and the conclusion follows.

Corollary 2.10. Consider the autonomous scalar FDE on $C$,

$$
\dot{x}(t)=b_{0} x(t)\left[1-L\left(x_{t}\right)\right],
$$

where $b_{0}>0$ and $L: C \rightarrow \mathbb{R}$ is a non-zero positive linear operator, and assume that $L$ satisfies (H2). Then all solutions $x(\varphi)(t)$ of (2.13) with initial conditions $\varphi$ as in (2.3) tend to $x_{*}$ as $t \rightarrow \infty$, where $x_{*}=l^{-1}$. Furthermore, $x_{*}$ is the only global solution of (2.13) that is bounded below from zero and bounded on $\mathbb{R}$.

EXAMPLE 2.11. Consider the following scalar delayed differential equation with a finite number of delays,

$$
\dot{x}(t)=x(t)\left[\alpha-b_{0} x(t)-\sum_{i=1}^{n} b_{i} x\left(t-r_{i}\right)\right],
$$

where all the constants $\alpha b_{i}, r_{i}(i=0, \ldots, n)$ are positive. If

$$
b_{0}>\sum_{i=1}^{n} b_{i}
$$

then (H1) and (2.10) hold, and $x_{*}=\alpha\left(\sum_{i=0}^{n} b_{i}\right)^{-1}$ is a globally stable equilibrium. From theorem 2.9, $x_{*}$ is the unique global solution of (2.14) that is bounded below from zero and bounded. We point out that the result on the global stability of $x_{*}$ was obtained in [8] without the restriction $b_{i}>0$, assuming (2.15) and the additional condition $b_{0} \geqslant \sum_{i=1}^{n}\left|b_{i}\right|$ (see also [6, theorem 5.6 and Remark 5.1, p. 35] for a correction of the proof in [8]). The results in both [8] and [6] were proven by using Lyapunov functional techniques. Further comments and remarks on equations in the form $(2.14)$ can be found in $[3, \S 5]$. 


\section{Non-autonomous linearity and asymptotic behaviour}

Consider a non-autonomous delayed logistic equation on $C$ of the form

$$
\dot{x}(t)=b(t) x(t)\left[a(t)-L\left(t, x_{t}\right)\right],
$$

where the following hold.

(A1) $a: \mathbb{R} \rightarrow \mathbb{R}, b: \mathbb{R} \rightarrow \mathbb{R}, L: \mathbb{R} \times C \rightarrow \mathbb{R}$ are continuous functions, and there are constants $\beta_{0}, \beta^{0}$ such that $0<\beta_{0} \leqslant b(t) \leqslant \beta^{0}$ for all $t \in \mathbb{R}$.

(A2) For all $t \in \mathbb{R}, L(t, \cdot)$ is a positive linear operator.

(A3) For all $M>0$, the function $(t, \varphi) \mapsto a(t)-L(t, \varphi)$ is uniformly bounded for $(t, \varphi) \in \mathbb{R} \times C,|\varphi|_{C} \leqslant M$.

Throughout this section, we assume (A1)-(A3) and consider solutions $x(\varphi)(t)$ of (3.1) with initial conditions

$$
x_{0}=\varphi,
$$

where $\varphi \in C$ is admissible, i.e. as in (2.3).

REMARK 3.1. Assumption (A3) requires that $a(t)$ be bounded on $\mathbb{R}$ and that $L(t, \cdot)$ be uniformly bounded in norm for all $t \in \mathbb{R}$, i.e. there exists $c_{0}>0$ such that

$$
\|L(t, \cdot)\| \leqslant c_{0} \quad \text { for all } t \in \mathbb{R} .
$$

In fact, these bound conditions follow immediately from noting that one can write $a(t)=a(t)-L(t, 0)$ and $L(t, \varphi)=a(t)-(a(t)-L(t, \varphi))$.

Lemma 3.2. Assume (A1)-(A3). The solution $x(\varphi)(t)$ of (3.1), (3.2) is positive and defined on $[0, \infty)$.

Proof. The proof follows by using standard arguments (cf. [12]).

In the sequel, we shall consider some additional hypotheses.

(A4) There is $\alpha_{0}>0$ such that

$$
L(t, 1)=\|L(t, \cdot)\| \geqslant \alpha_{0} \quad \text { for all } t \in \mathbb{R} .
$$

(A5) For all $\varphi \in C$, there exists $\lim _{t \rightarrow \infty} L(t, \varphi):=L(\varphi)$.

We observe that under (A1)-(A3) and (A5), the limit operator $L$ is also linear and positive with $l:=\|L\| \leqslant c_{0}$, where $c_{0}$ is as in (3.3).

TheOREm 3.3. Assume (A1)-(A4) and that, for some $\delta$,

$$
a(t) \geqslant \delta>0 \quad \text { for } t \text { large. }
$$

Then the solutions $x(\varphi)(t)$ of the IVPs (3.1), (3.2) are defined, bounded below from zero and bounded on $[0, \infty)$. 
Proof. For $x(t)=x(\varphi)(t), t \geqslant 0$, we have $\dot{x}(t) \leqslant \gamma(t) x(t)$, where $\gamma(t)=a(t) b(t)$. Therefore,

$$
x\left(t_{0}\right) \geqslant x(t) \exp \left(-\int_{t_{0}}^{t} \gamma(s) \mathrm{d} s\right), \quad 0 \leqslant t_{0} \leqslant t .
$$

From remark 3.1 and $(\mathrm{A} 1), \gamma(t)$ is bounded on $\mathbb{R}$. Consider $m \in \mathbb{R}$ such that

$$
\sup _{\theta \in[-r, 0]} \int_{t+\theta}^{t} \gamma(s) \mathrm{d} s \leqslant m, \quad t \geqslant 0 .
$$

Hence $x_{t}(\theta) \geqslant x(t) \mathrm{e}^{-m},-r \leqslant \theta \leqslant 0$, which implies

$$
\dot{x}(t) \leqslant x(t)[\gamma(t)-g(t) x(t)]
$$

where $g(t)=b(t) L(t, 1) \mathrm{e}^{-m}$ is continuous and satisfies

$$
0<\alpha_{0} \beta_{0} e^{-m} \leqslant g(t) \leqslant c_{0} \beta^{0} \mathrm{e}^{-m}, \quad t \in \mathbb{R}
$$

with $\alpha_{0}$ and $c_{0}$ as in equations (3.4) and (3.3), respectively. Note that the function $f(t, y)=\gamma(t)-g(t) y$ is continuous and that $\partial f / \partial y \leqslant 0$ on $\mathbb{R}^{2}$. It follows (see, for example, [13, theorem 1]) that all solutions of the scalar ODE

$$
\dot{y}(t)=y(t)[\gamma(t)-g(t) y(t)]
$$

with a positive initial condition at $t=0$ are defined and bounded on $[0, \infty)$. By a comparison result applied to (3.6), we conclude that $x(t)$ is bounded on $[0, \infty)$. Since $x(t)$ is bounded on $[-r, \infty)$, from (A3), let $K>0$ be such $a(t)-L\left(t, x_{t}\right) \geqslant-K$ for $t \geqslant 0$. It follows that $\dot{x}(t) \geqslant-K \beta^{0} x(t), t \geqslant 0$. Hence $x_{t}(\theta) \leqslant x(t) \mathrm{e}^{K r \beta^{0}}$ for $\theta \in[-r, 0]$ and $t \geqslant r$. Define

$$
h(t)=\mathrm{e}^{K r \beta^{0}} b(t) L(t, 1) .
$$

We get $\dot{x}(t) \geqslant x(t)[\gamma(t)-h(t) x(t)]$. On the other hand, equation (3.5) implies that $\gamma(t) \geqslant \delta \beta_{0}>0$ for $t$ large. Thus all the solutions of the logistic ODE

$$
\dot{y}(t)=y(t)[\gamma(t)-h(t) y(t)]
$$

with initial conditions $y(0)>0$ are bounded below from zero on $[0, \infty)$ (see $[13$, theorem 2]). Hence $x(t)$ is also bounded below from zero on $[0, \infty)$.

The next results concern the asymptotic behaviour of solutions of (3.1), (3.2).

Theorem 3.4. Assume (A1)-(A5). Assume also that the following hold.

(i) $a(t) \rightarrow a_{0}>0$ as $t \rightarrow \infty$.

(ii) The operator L in (A5) satisfies (H2). Then

$$
\lim _{t \rightarrow \infty} x(t)=x_{*}
$$

where $x(t)=x(\varphi)(t)$ is the solution of the IVP (3.1), (3.2), and $x_{*}=a_{0} /\|L\|$. 
Proof. The proof follows by adjusting the technique used to prove theorem 2.3 . Let $x(t)$ be the solution of (3.1), (3.2), and effect the change of variables $y=\left(x-x_{*}\right) / x_{*}$. Then $y(t)$ is a solution of

$$
\dot{y}(t)=b(t)(1+y(t))\left[a(t)-x_{*} L(t, 1)-x_{*} L\left(t, y_{t}\right)\right] .
$$

First, we consider the case of $y(t)$ eventually monotone. Let $c=\lim _{t \rightarrow \infty} y(t)$ and suppose that $c>0$. The case $c<0$ is analogous. Fix $\varepsilon>0$ small with

$$
c-\varepsilon>0, \quad \varepsilon+a_{0}[\varepsilon-(c-\varepsilon)(1-\varepsilon)]<0 .
$$

From (i), (A5) and the definition of $c$, there is $T_{1}$ such that

$$
\begin{aligned}
& y(t) \geqslant c-\varepsilon \quad \text { for } t \geqslant T_{1}-r, \\
& a(t) \leqslant a_{0}+\varepsilon, \quad L(t, 1) \geqslant\|L\|(1-\varepsilon) \quad \text { for } t \geqslant T_{1} .
\end{aligned}
$$

Then, for $t \geqslant T_{1}$, we obtain

$$
\begin{aligned}
1+y(t) & =\left(1+y\left(T_{1}\right)\right) \exp \left(\int_{T_{1}}^{t} b(s)\left[a(s)-x_{*} L(s, 1)-x_{*} L\left(s, y_{s}\right)\right] \mathrm{d} s\right) \\
& \leqslant\left(1+y\left(T_{1}\right)\right) \exp \left(\beta_{0}\left\{\varepsilon+a_{0}[\varepsilon-(c-\varepsilon)(1-\varepsilon)]\right\}\left(t-T_{1}\right)\right) \\
& \rightarrow 0 \quad \text { as } t \rightarrow \infty,
\end{aligned}
$$

which is a contradiction. Hence $c=0$.

Now consider the case of $y(t)$ not eventually monotone. Define

$$
\liminf _{t \rightarrow \infty} y(t)=-v, \quad \limsup _{t \rightarrow \infty} y(t)=u .
$$

Note that theorem 3.3 implies that $-1<-v \leqslant u<\infty$. Fix $\varepsilon>0$ and let $T>0$ be such that

$$
-v-\varepsilon \leqslant y(t) \leqslant u+\varepsilon, \quad t>T .
$$

Consider the case $|v| \leqslant u$. Let $\left(t_{n}\right)$ be a sequence such that $t_{n}-r>T, \dot{y}\left(t_{n}\right)=0$, $y\left(t_{n}\right)>0, y\left(t_{n}\right) \rightarrow u$ and $t_{n} \rightarrow \infty$ as $n \rightarrow \infty$. From (3.7), we get

$$
a\left(t_{n}\right)-x_{*} L\left(t_{n}, 1\right)-x_{*} L\left(t_{n}, y_{t_{n}}\right)=0 .
$$

Clearly, from theorem 3.3, it follows that both $y(t)$ and $\dot{y}(t)$ are uniformly bounded on $[0, \infty)$. Thus $y(t)$ is uniformly continuous on $[0, \infty)$. This implies that the sequence $\left(y_{t_{n}}\right) \subset C$ is uniformly bounded and equicontinuous. Therefore, there exists a subsequence, still denoted by $\left(y_{t_{n}}\right)$, and $\varphi \in C$ such that $\left(y_{t_{n}}\right)$ converges to $\varphi$ on $C$. Since $y_{t_{n}}(0) \rightarrow u, n \rightarrow \infty$, then $\varphi(0)=u$. On the other hand, from (3.7), (i), (3.3) and (A5), we have

$$
\begin{aligned}
0 & =a\left(t_{n}\right)-x_{*} L\left(t_{n}, 1\right)-x_{*} L\left(t_{n}, y_{t_{n}}\right) \\
& =\left[a\left(t_{n}\right)-x_{*} L\left(t_{n}, 1\right)\right]-x_{*}\left[L\left(t_{n}, y_{t_{n}}\right)-L\left(t_{n}, \varphi\right)\right]-x_{*} L\left(t_{n}, \varphi\right) \\
& \rightarrow-x_{*} L(\varphi),
\end{aligned}
$$

and we conclude that $L(\varphi)=0$. From (3.8) and $|v| \leqslant u$, we get $\left|y_{t_{n}}\right|_{C} \leqslant(u+\varepsilon)$. Since $\varepsilon>0$ is arbitrary, by letting $n \rightarrow \infty$, we obtain $|\varphi|_{C} \leqslant u=\varphi(0)$. But $L$ satisfies hypothesis (H2). Therefore, we deduce that $u=0$. The assumption 
$|v| \leqslant u$ now implies that $v=0$, and the conclusion follows. The case $|u| \leqslant v$ is analogous.

A particular case of (3.1) under hypotheses (A1)-(A5) is considered in the following result.

Corollary 3.5. Consider the scalar FDE on $C$,

$$
\dot{x}(t)=x(t)\left[a(t)-h(t) L\left(x_{t}\right)\right],
$$

where $a: \mathbb{R} \rightarrow \mathbb{R}, h: \mathbb{R} \rightarrow \mathbb{R}$ are continuous, $L: C \rightarrow \mathbb{R}$ is a non-zero positive linear operator and there are constants $\alpha_{0}, \alpha^{0}, \beta_{0}, \beta^{0}$ such that $\alpha_{0} \leqslant a(t) \leqslant \alpha^{0}$ and $0<\beta_{0} \leqslant h(t) \leqslant \beta^{0}$ for all $t \in \mathbb{R}$. Then all the solutions $x(\varphi)(t)$ of the IVPs (3.9), (3.2) are bounded below from zero and bounded on $[0, \infty)$. Furthermore, if $a(t) \rightarrow a_{0}>0, h(t) \rightarrow h_{0}$ as $t \rightarrow \infty$, and $L$ satisfies (H2), then

$$
\lim _{t \rightarrow \infty} x(\varphi)(t)=x_{*},
$$

where $x_{*}=a_{0} /\left(h_{0}\|L\|\right)$.

EXAMPLE 3.6. As an application of the previous results, consider a model with discrete delays,

$$
\dot{x}(t)=b(t) x(t)\left[a(t)-\alpha_{0}(t) x(t)-\sum_{i=1}^{n} \alpha_{i}(t) x\left(t-r_{i}\right)\right],
$$

where $r_{i}>0, a, b, \alpha_{0}, \alpha_{i}: \mathbb{R} \rightarrow \mathbb{R}$ are continuous and bounded, $0<\beta_{0} \leqslant b(t) \leqslant \beta^{0}$, and the functions $\alpha_{0}, \alpha_{i}$ are non-negative, $i=1, \ldots, n$. Suppose that $a(t) \rightarrow a_{0}>0$ and $\alpha_{i}(t) \rightarrow c_{i}$ as $t \rightarrow \infty, i=0, \ldots, n, c_{0}>0$. If $c_{0}>\sum_{i=1}^{n} c_{i}$, from theorem 3.4, we conclude that the solutions of (3.10) with admissible initial conditions tend to $x_{*}=a_{0}\left(\sum_{i=0}^{n} c_{i}\right)^{-1}$ as $t$ goes to $\infty$.

ExAmPle 3.7. Consider the scalar model

$$
\dot{x}(t)=b(t) x(t)\left[a(t)-\alpha(t) x(t)-c(t) \int_{-r}^{0} K(\theta) x(t+\theta) \mathrm{d} \theta\right],
$$

where $r>0, a, \alpha, b, c: \mathbb{R} \rightarrow \mathbb{R}$ are continuous and bounded, $0<\beta_{0} \leqslant b(t) \leqslant \beta^{0}$, $\alpha(t), c(t) \geqslant 0$ for all $t \in \mathbb{R}$ and $K:[-r, 0] \rightarrow[0, \infty)$ is integrable.

Corollary 3.8. Assume that the following hold.

(i) $a(t) \rightarrow a_{0}>0, \alpha(t) \rightarrow \alpha_{0}>0, c(t) \rightarrow c_{0}$ as $t \rightarrow \infty$.

(ii) $c_{0} \int_{-r}^{0} K(\theta) \mathrm{d} \theta \leqslant \alpha_{0}$.

(iii) If $c_{0} \int_{-r}^{0} K(\theta) \mathrm{d} \theta=\alpha_{0}$, there exists $\varepsilon \in(-r, 0)$ such that $\int_{\varepsilon}^{0} K(\theta) \mathrm{d} \theta>0$.

Then the solutions $x(t)=x(\varphi)(t)$ of the IVPs (3.11), (3.2) satisfy

$$
x(t) \rightarrow a_{0}\left(\alpha_{0}+c_{0} \int_{-r}^{0} K(\theta) \mathrm{d} \theta\right)^{-1} \text { as } t \rightarrow \infty .
$$


Proof. For $\varphi \in C$, define

$$
\begin{aligned}
L(t, \varphi) & =\alpha(t) \varphi(0)+c(t) \int_{-r}^{0} K(\theta) \varphi(\theta) \mathrm{d} \theta, \\
L(\varphi) & =\alpha_{0} \varphi(0)+c_{0} \int_{-r}^{0} K(\theta) \varphi(\theta) \mathrm{d} \theta .
\end{aligned}
$$

Clearly, assumptions (A1)-(A3) hold and $L(t, \varphi) \rightarrow L(\varphi)$ as $t$ tends to $\infty$ for all $\varphi \in C$. Since $L(t, 1) \geqslant \alpha(t) \rightarrow \alpha_{0}>0$ as $t \rightarrow \infty$, assumption (A4) is fulfilled. From (ii) and (iii), we conclude that $L$ satisfies (H2) (cf. remark 2.4 and example 2.5). The result follows now from theorem 3.4.

In an obvious way, similar statements can be given for more general models of the type

$$
\dot{x}(t)=b(t) x(t)\left[a(t)-\alpha(t) x(t)-\sum_{i=1}^{n} \alpha_{i}(t) \int_{-r}^{0} K_{i}(\theta) x(t+\theta) \mathrm{d} \theta\right] .
$$

\section{Boundedness and stability for the case of an autonomous non-positive linearity}

In this section, we will apply some techniques employed in $\S 2$ to address the boundedness of positive solutions and the global stability of the positive equilibrium of (2.1), when it exists, for the general case of $L: C \rightarrow \mathbb{R}$ a non-zero linear bounded operator not necessarily positive.

Consider (2.1), and let $\eta:[-r, 0] \rightarrow \mathbb{R}$ be a bounded variation function such that

$$
L(\varphi)=\int_{-r}^{0} \varphi(\theta) \mathrm{d} \eta(\theta), \quad \varphi \in C .
$$

It is always possible to decompose $\eta$ as $\eta=\eta_{1}-\eta_{2}$, where $\eta_{i}:[-r, 0] \rightarrow \mathbb{R}$ are non-decreasing, $i=1,2$. Thus $L$ is written as $L(\varphi)=L_{1}(\varphi)-L_{2}(\varphi)$, where $L_{i}(\varphi)=\int_{-r}^{0} \varphi(\theta) \mathrm{d} \eta_{i}(\theta), i=1,2$, are linear and positive. Equation (2.1) becomes

$$
\dot{x}(t)=b(t) x(t)\left[1-L_{1}\left(x_{t}\right)+L_{2}\left(x_{t}\right)\right] .
$$

Note that (4.1) has a positive equilibrium given by

$$
x_{*}=1 /\left(\left\|L_{1}\right\|-\left\|L_{2}\right\|\right)
$$

if and only if $\left\|L_{1}\right\|>\left\|L_{2}\right\|$. Clearly, this is the case if (H2) holds, since (H2) implies that $L(1)=\left\|L_{1}\right\|-\left\|L_{2}\right\|>0$.

As in the previous sections, we only consider admissible initial conditions $x_{0}=\varphi$ such that $\varphi(\theta) \geqslant 0, \theta \in[-r, 0), \varphi(0)>0$. Consequently, the solution $x(\varphi)(t)$ of (4.1), (2.2) is positive for $t \geqslant 0$ on its maximal existence interval.

Theorem 4.1. Let $L=L_{1}-L_{2}$, where $L_{1}, L_{2}$ are positive linear operators. Assume that hypotheses (H1) (i) and (H2) are satisfied. Then the solutions $x(t)=x(\varphi)(t)$ of (4.1) are defined, bounded below from zero and bounded on $[0, \infty)$. Moreover, $\lim _{t \rightarrow \infty} x(t)=x_{*}$, where $x_{*}=1 /\left(\left\|L_{1}\right\|-\left\|L_{2}\right\|\right)$ is the unique positive equilibrium of (4.1). 
Proof. Let $x(t)=x(\varphi)(t)$ be the solution of (4.1), (2.2) defined on $I:=[-r, a)$ $(a \in \mathbb{R}$ or $a=\infty)$.

Fix $t_{0} \in[0, a)$ and define

$$
K=\max _{-r \leqslant t \leqslant t_{0}}\left|x(t)-x_{*}\right|,
$$

where $x_{*}=1 /\left(\left\|L_{1}\right\|-\left\|L_{2}\right\|\right)$. By repeating the same arguments as in the proof of lemma 2.6, we conclude that

$$
\left|x(t)-x_{*}\right| \leqslant K \quad \text { for } t \in I .
$$

Hence $x(t)$ is defined for all $t \geqslant 0$. Since $x(t)$ is bounded, standard arguments show that $x(t)$ is also bounded below from zero.

By the change of variables $y=\left(x-x_{*}\right) / x_{*}, y(t)$ is a solution of

$$
\dot{y}(t)=-x_{*} b(t)(1+y(t)) L\left(y_{t}\right) .
$$

To prove that $y(t) \rightarrow 0$ as $t \rightarrow \infty$, we consider two cases.

CASE 1. Suppose that $y(t)$ is eventually monotone.

Let $c=\lim _{t \rightarrow \infty} y(t)$. Suppose that $c \geqslant 0$ (the situation $c \leqslant 0$ is treated in a similar way). In order to get a contradiction, let $c>0$. Fix $\varepsilon>0$ such that $\varepsilon<c\left(\left\|L_{1}\right\|-\left\|L_{2}\right\|\right) /\left(\left\|L_{1}\right\|+\left\|L_{2}\right\|\right) \leqslant c$, and choose $T_{1}$ such that

$$
c-\varepsilon \leqslant y_{t} \leqslant c+\varepsilon, \quad t \geqslant T_{1} .
$$

From (4.2), one gets

$$
1+y(t)=\left(1+y\left(T_{1}\right)\right) \exp \left(-x_{*} \int_{T_{1}}^{t} b(s) L\left(y_{s}\right) \mathrm{d} s\right), \quad t \geqslant T_{1} .
$$

On the other hand, from (4.3) and since $\left\|L_{1}\right\|>\left\|L_{2}\right\|$, for $t \geqslant T_{1}$, we have

$$
\begin{aligned}
L\left(y_{t}\right) & =L_{1}\left(y_{t}\right)-L_{2}\left(y_{t}\right) \\
& \geqslant(c-\varepsilon)\left\|L_{1}\right\|-(c+\varepsilon)\left\|L_{2}\right\| \\
& =c\left(\left\|L_{1}\right\|-\left\|L_{2}\right\|\right)-\varepsilon\left(\left\|L_{1}\right\|+\left\|L_{2}\right\|\right) \\
& :=c_{1}>0 .
\end{aligned}
$$

From (4.4), we deduce that

$$
1+y(t) \leqslant\left(1+y\left(T_{1}\right)\right) \mathrm{e}^{-x_{*} \beta_{0} c_{1}\left(t-T_{1}\right)}, \quad t \geqslant T_{1} .
$$

By letting $t \rightarrow \infty$, we obtain $1+c \leqslant 0$, which is a contradiction.

CAse 2. $y(t)$ is not eventually monotone.

The proof that $\lim _{t \rightarrow \infty} y(t)=0$ follows by using the same arguments as in the proof of theorem 2.3, so we omit it.

The next statements refer to a particular case of (4.1), when $L$ has a non-delayed term. 
TheOREM 4.2. Assume (H1) (i) and let

$$
L(\varphi)=b_{0} \varphi(0)+\tilde{L}_{1}(\varphi)-L_{2}(\varphi), \quad \varphi \in C,
$$

where $b_{0}>0$ and $\tilde{L}_{1}, L_{2}$ are positive linear operators. If $b_{0}>\left\|L_{2}\right\|$, then the solution of (4.1), (2.2) is defined, bounded below from zero and bounded for $t \geqslant 0$.

Proof. Let $x(t)=x(\varphi)(t)$ be the solution of (4.1), (2.2) defined on $I:=[-r, a)$ $(a \in \mathbb{R}$ or $a=\infty)$. We claim that $x(t)<M$ for $t \in I$, where

$$
M>\max \left\{|\varphi|_{C},\left(b_{0}-\| L_{2}||\right)^{-1}\right\} \text {. }
$$

Otherwise, there exists $T_{1}>0$ such that $0 \leqslant x(t)<M$ for $t \in\left[-r, T_{1}\right)$ and $x\left(T_{1}\right)=M$. Thus $\dot{x}\left(T_{1}\right) \geqslant 0$ and, by $(4.1)$,

$$
\dot{x}\left(T_{1}\right) \leqslant b\left(T_{1}\right) x\left(T_{1}\right)\left[1-b_{0} x\left(T_{1}\right)+L_{2}\left(x_{T_{1}}\right)\right] \leqslant b\left(T_{1}\right) x\left(T_{1}\right)\left[1-\left(b_{0}-\left\|L_{2}\right\|\right) M\right]<0
$$

a contradiction. Hence $x(t)$ is defined and bounded for $t \geqslant 0$. This implies that $x(t)$ is also bounded below from zero on $[0, \infty)$.

Corollary 4.3. Assume (H1) (i) and let L have the form (4.5), where $b_{0}>0$ and $\tilde{L}_{1}, L_{2}$ are positive linear operators. If (H2) is satisfied, then the solution of (4.1), (2.2) is defined, bounded below from zero and bounded for $t \geqslant 0$, and satisfies $\lim _{t \rightarrow \infty} x(t)=x_{*}$, where $x_{*}=\left(b_{0}+\left\|\tilde{L}_{1}\right\|-\left\|L_{2}\right\|\right)^{-1}$.

Proof. The result follows from theorem 4.1. Note that, in particular, hypothesis (H2) implies

$$
b_{0}+\left\|\tilde{L}_{1}\right\|>\left\|L_{2}\right\|
$$

It is important to recall that the situation $\tilde{L}_{1}=0$ is included in theorem 4.2 and corollary 4.3 .

ExAmPLE 4.4. Consider the general scalar Lotka-Volterra equation with distributed delays

$$
\dot{x}(t)=\beta(t) x(t)\left[\gamma-a x(t)+b \int_{-r}^{0} x(t+\theta) \mathrm{d} \mu_{1}(\theta)-c \int_{-r}^{0} x(t+\theta) \mathrm{d} \mu_{2}(\theta)\right],
$$

where $\beta: \mathbb{R} \rightarrow \mathbb{R}$ is continuous, $0<\beta_{0} \leqslant \beta(t) \leqslant \beta^{0}$ for $t \in \mathbb{R}, \gamma, a, b$, c are non-negative constants and $\mu_{i}$ are non-decreasing, $\int_{-r}^{0} \mathrm{~d} \mu_{i}(\theta)=1, i=1,2$. Equation (4.7) with $\beta(t) \equiv 1$ was investigated by Kuang [6, example 5.3, pp. 34-37].

Denote $L(\varphi)=a \varphi(0)+L_{0}(\varphi)$, for

$$
L_{0}(\varphi)=c \int_{-r}^{0} \varphi(\theta) \mathrm{d} \mu_{2}(\theta)-b \int_{-r}^{0} \varphi(\theta) \mathrm{d} \mu_{1}(\theta) .
$$

Corollary 4.3 states that if $L$ satisfies (H2), then all solutions of (4.7) with admissible initial conditions are defined for $t \geqslant 0$ and converge to $x_{*}=\gamma /(a+c-b)$ as $t \rightarrow \infty$.

Consider the case $\beta(t) \equiv 1$. Kuang obtained the global asymptotic stability of $x_{*}$ under the conditions

$$
b+c \leqslant a, \quad b-c<a .
$$


For $c=0$, Kuang's result and our corollary 4.3 give the same conclusion (see (4.6)). Now let $c>0$. It is clear that $\left\|L_{0}\right\| \leqslant b+c$. Assume that $\left\|L_{0}\right\|<b+c$. In this situation, Kuang's theorem fails for $a<b+c$, while corollary 4.3 allows us to assure that $x_{*}$ is globally asymptotically stable for all $a>\left\|L_{0}\right\|$, since in this case $(2.10)$ is fulfilled with $d=\left\|L_{0}\right\| / a<1$.

On the other hand, if $\mu_{2}$ is such that there is $\varepsilon \in(0, r)$ with $\mu_{2}(\theta)<\mu_{2}(0)$ for $-\varepsilon<\theta<0$, then condition $b+c \leqslant a$ implies (H2). In fact, consider $\varphi \in C$ with $|\varphi|_{C}=\varphi(0)>0$. If $b+c<a$, clearly $L(\varphi) \geqslant a \varphi(0)-(b+c)|\varphi|_{C}>0$. If $b+c=a$, then

$$
\begin{aligned}
L(\varphi) & =c \varphi(0)+c \int_{-r}^{0} \varphi(\theta) \mathrm{d} \mu_{2}(\theta)+b \int_{-r}^{0}[\varphi(0)-\varphi(\theta)] \mathrm{d} \mu_{1}(\theta) \\
& \geqslant c \varphi(0)+c \int_{-r}^{0} \varphi(\theta) \mathrm{d} \mu_{2}(\theta) .
\end{aligned}
$$

Arguing as in example 2.5, we conclude that $L(\varphi)>0$, and hence (H2) holds.

REMARK 4.5. It would be interesting to find new conditions for the boundedness of positive solutions of (4.1) for the case of any bounded linear operator $L$. In general, the condition $\left\|L_{1}\right\|>\left\|L_{2}\right\|$, where $L=L_{1}-L_{2}, L_{1}, L_{2}$ positive, is not sufficient to guarantee that the solutions of (4.1) with admissible initial conditions are defined and bounded for $t \geqslant 0$ (see [3, problem 5.2]). However, here we were able to prove this result under the stronger condition $(\mathrm{H} 2)$.

\section{Acknowledgments}

This work was partly supported by FCT (Portugal) under project POCTI/32931/ MAT/2000 (T.F.) and MCT (Spain) and FEDER under project BFM2001-3884 (E.L.).

The paper was partly written while E.L. was visiting the Centro de Matemática e Aplicações Fundamentais (CMAF) of the University of Lisbon. It is his pleasure to thank the University for its kind hospitality.

The authors thank C. Martínez for helpful discussions.

\section{References}

1 M. P. Chen, J. S. Yu, X. Z. Qian and Z. C. Wang. On the stability of a delay differential population model. Nonlin. Analysis 25 (1995), 187-195.

2 K. Gopalsamy. Stability and oscillation in delay differential equations of population dymam ics (Dordrecht: Kluwer Academic Publishers, 1992).

3 I. Györi. A new approach to the global stability problem in a delay Lotka-Volterra differential equation. Math. Comput. Modelling 31 (2000), 9-28.

4 J. R. Haddock and Y. Kuang. Asymptotic theory for a class of nonautonomous delay differential equations. J. Math. Analysis Applic. 168 (1992), 147-162.

5 Y. Kuang. Global stability for a class of nonlinear nonautonomous delay equations. Nonlin. Analysis 17 (1991), 627-634.

6 Y. Kuang. Delay differential equations with applications in population dymamics (Academic, 1993).

$7 \quad$ Y. Kuang and H. L. Smith. Global stability for infinite delay Lotka-Volterra type systems. J. Diff. Eqns 103 (1993), 221-246. 
8 S. M. Lenhart and C. C. Travis. Global stability of a biological model with time delay. Proc. Am. Math. Soc. 96 (1986), 75-78.

9 R. K. Miller. On Volterra's population equation. SIAM J. Appl. Math. 14 (1966), 446-452.

10 G. Seifert. On a delay-differential equation for single specie population variations. Nonlin. Analysis 11 (1987), 1051-1059.

11 J. W.-H. So and J. S. Yu. Global attractivity for a population model with time delay. Proc. Am. Math. Soc. 123 (1995), 2687-2694.

12 J. W.-H. So and J. S. Yu. Global stability for a general population model with time delays. Fields Inst. Common. 21 (1999), 447-457.

13 R. R. Vance and E. A. Coddington. A nonautonomous model of population growth. J. Math. Biol. 27 (1989), 491-506.

14 E. M. Wright. A non-linear difference-differential equation. J. Reine Angew. Math. 194 $(1955), 66-87$.

(Issued 31 October 2003) 